\title{
BMJ Open Shunting outcomes in communicating hydrocephalus: protocol for a multicentre, open-label, randomised controlled trial
}

\author{
Tong Sun, ${ }^{1}$ Wenyao Cui, ${ }^{1}$ Jingguo Yang, ${ }^{1}$ Yikai Yuan, ${ }^{1}$ Xuepei $\mathrm{Li},{ }^{2} \mathrm{Hang} \mathrm{Yu},{ }^{3}$ \\ Yicheng Zhou, ${ }^{1}$ Chao You, ${ }^{1,4}$ Junwen Guan (D) ${ }^{1}$
}

To cite: Sun T, Cui W, Yang J, et al. Shunting outcomes in communicating hydrocephalus: protocol for a multicentre, open-label, randomised controlled trial. BMJ Open 2021;11:e051127. doi:10.1136/ bmjopen-2021-051127

- Prepublication history and additional supplemental material for this paper are available online. To view these files, please visit the journal online (http://dx.doi.org/10.1136/ bmjopen-2021-051127)

TS and WC contributed equally.

Received 10 March 2021 Accepted 17 August 2021

Check for updates

(C) Author(s) (or their employer(s)) 2021. Re-use permitted under CC BY-NC. No commercial re-use. See rights and permissions. Published by BMJ.

${ }^{1}$ Department of Neurosurgery, Sichuan University West China Hospital, Chengdu, Sichuan, China

${ }^{2}$ Medical Simulation Center, Chengdu First People's Hospital, Chengdu, Sichuan, China

${ }^{3}$ Department of Neurology, Sichuan Provincial People's Hospital, Chengdu, Sichuan, China

${ }^{4}$ Neurosurgery Research Laboratory, Sichuan University West China Hospital, Chengdu, Sichuan, China

Correspondence to

Dr Junwen Guan;

guanjunwen_scu@outlook.com

\section{ABSTRACT}

Introduction Ventriculoperitoneal shunt (VPS) remains the most widely used methods to treat communicating hydrocephalus. More recently, lumboperitoneal shunt (LPS) has been suggested as a reasonable option in some studies. However, there is lack of high-quality studies comparing these two techniques in order to certain the benefits and harms to use one of these two methods. The purpose of the current study is to determine the effectiveness and safety of the LPS versus the VPS in patients with communicating hydrocephalus. Methods and analysis All eligible patients aged 18-90 years with communicating hydrocephalus will be recruited and then randomly allocated into LPS or VPS group in a ratio of 1:1. All patients will be analysed before shunt insertion, at the time of discharge, 1 month, 6 months, 12 months and 24 months postoperatively. The primary outcome measure is the rate of shunt failure at a 2-year follow-up term. The secondary outcomes include Keifer's Hydrocephalus Scale, National Institute of Health Stroke Scale, Glasgow Outcome Scale Extended, Evans index, safety endpoints and cost-effectiveness of hospital stay.

Ethics and dissemination The study will be performed in compliance with the Declaration of Helsinki (2002) of the World Medical Association. The study was approved by Institutional Review Board of West China Hospital. All patients will be fully informed the potential benefits, potential risks and responsibilities, those who will sign the informed consents once they are included. Preliminary and final results will be published in peer-reviewed journals and presented at national and international congresses.

Trial registration number ChiCTR2100043839.

\section{INTRODUCTION}

Communicating hydrocephalus, a common type of hydrocephalus, is the pathological process where ventricles enlarged, progressively compressing periventricular white matter. ${ }^{1-3}$ Intracerebral haemorrhage (ICH) is one of common risk factors for communicating hydrocephalus, along with traumatic brain injury and intracranial infection. ${ }^{4}$ Some elderly population to develop communicating hydrocephalus without any known causes are probably diagnosed as idiopathic normal-pressure hydrocephalus (INPH). ${ }^{6}$

\section{Strengths and limitations of this study}

- This study is the first randomised controlled trial about comparing these two procedures of cerebrospinal fluid diversions in the treatment of communicating hydrocephalus.

- The current study will provide high-level evidence on the advantages and disadvantages of these two methods.

- This study will provide high-level evidence on the optimal option in the treatment of normal-pressure hydrocephalus based on a randomised controlled trial.

- This trial will help to create an algorithm for the selection of suitable patients, preshunt and postshunt management.

- Medical condition and experience of attending surgeons are sometimes various leading to potential bias but the neurosurgeons will be trained centrally in advance and reach uniform standard.

Different strategies for diverting cerebrospinal fluid (CSF) have long been proposed as standard treatments for communicating hydrocephalus. Ventriculoperitoneal shunt (VPS) remains the most widely used method to treat communicating hydrocephalus. ${ }^{7}$ Endoscopic third ventriculostomy (ETV) is an alternative and effective option for obstructive hydrocephalus, and has recently been performed for communicating types of hydrocephalus. ${ }^{49} \mathrm{~A}$ randomised controlled trial showed patients with INPH treated by ETV obtained worse neurological outcomes and higher incidence of severe complications than those who were treated with VPS. ${ }^{10}$ More recently, lumboperitoneal shunt (LPS) has been suggested as a reasonable option in some studies, though this is typically recommended when patients are diagnosed as INPH. ${ }^{11}$ For instance, LPS has become the superior option for patients with INPH over VPS in Japan. Some clinical trials also indicated LPS was safe and effective for other types of communicating hydrocephalus including posthaemorrhagic hydrocephalus and posttraumatic hydrocephalus. ${ }^{12-16}$ 
LPS has some advantages over VPS, including the lower risk of brain injury and lower incidence of infection. ${ }^{17}$ Despite potential advantages, the comparison of VPS to LPS in the treatment of communicating hydrocephalus is poorly understood. Currently, there are few prospective studies comparing the efficacy of these two techniques. Early evidence had ever revealed LPS was more likely to undergo shunt failure compared with VPS (7\% vs 1\%) while a recent retrospective study suggested patients with communicating hydrocephalus secondary to ICH treated by VPS or LPS had equivalent clinical results. ${ }^{14}{ }^{18}$ In addition, Giordan $e t ~ a l^{19}$ recently performed a systematic review and meta-analysis, suggesting the shunting outcomes did not differ significantly among different CSF diversion techniques used. However, there is lack of high-quality studies comparing these two techniques in order to certain the benefits and harms to use one of these two methods.

\section{Objective}

The purpose of the current study is to determine the effectiveness and safety of the LPS versus the VPS in patients with communicating hydrocephalus.

\section{METHODS AND ANALYSIS}

\section{Patient and public involvement}

No patient or public is involved in study design, recruitment or conduct of the study.

\section{Study design and settings}

The current study is a multicentre, open-label and randomised controlled trial in which 550 patients with communicating hydrocephalus will be randomly allocated into LPS or LPS group in a ratio of 1:1. Patients will be enrolled at 20 neurosurgical centres in China Mainland that are experienced and skilled in both neurosurgery and shunt surgery. Each participating site will receive the local ethics committee approval, or obey our ethics committee review decision. We will propose the standardised procedures for CSF diversion and perioperative management before enrolment, and every attending neurosurgeon will be trained centrally. All patients will be fully informed the potential benefits, potential risks and responsibilities, those who will sign the informed consents once they are included. This study protocol is developed following the Guidelines of Standard Protocol Items: Recommendations for Interventional Trials. ${ }^{20}$ The planned start date is September 2021 and end date is June 2028.

\section{Sample size}

A recent meta-analysis indicated the rate of VPS failure and LPS failure were $18.0 \%$ and $14.0 \%$, respectively. ${ }^{21}$ In this light, a sample of 250 for each group will be required in this trial while the significance level (two sided) is $5 \%$ and the test power is $80 \%$. Considering about the lost to follow-up within 2 years, the sample size is enlarged to 275 for each group.

\section{Recruitment and eligibility criteria}

Figure 1 shows the flow chart of the selection of patients. The enrolment is expected to commenced in September 2021
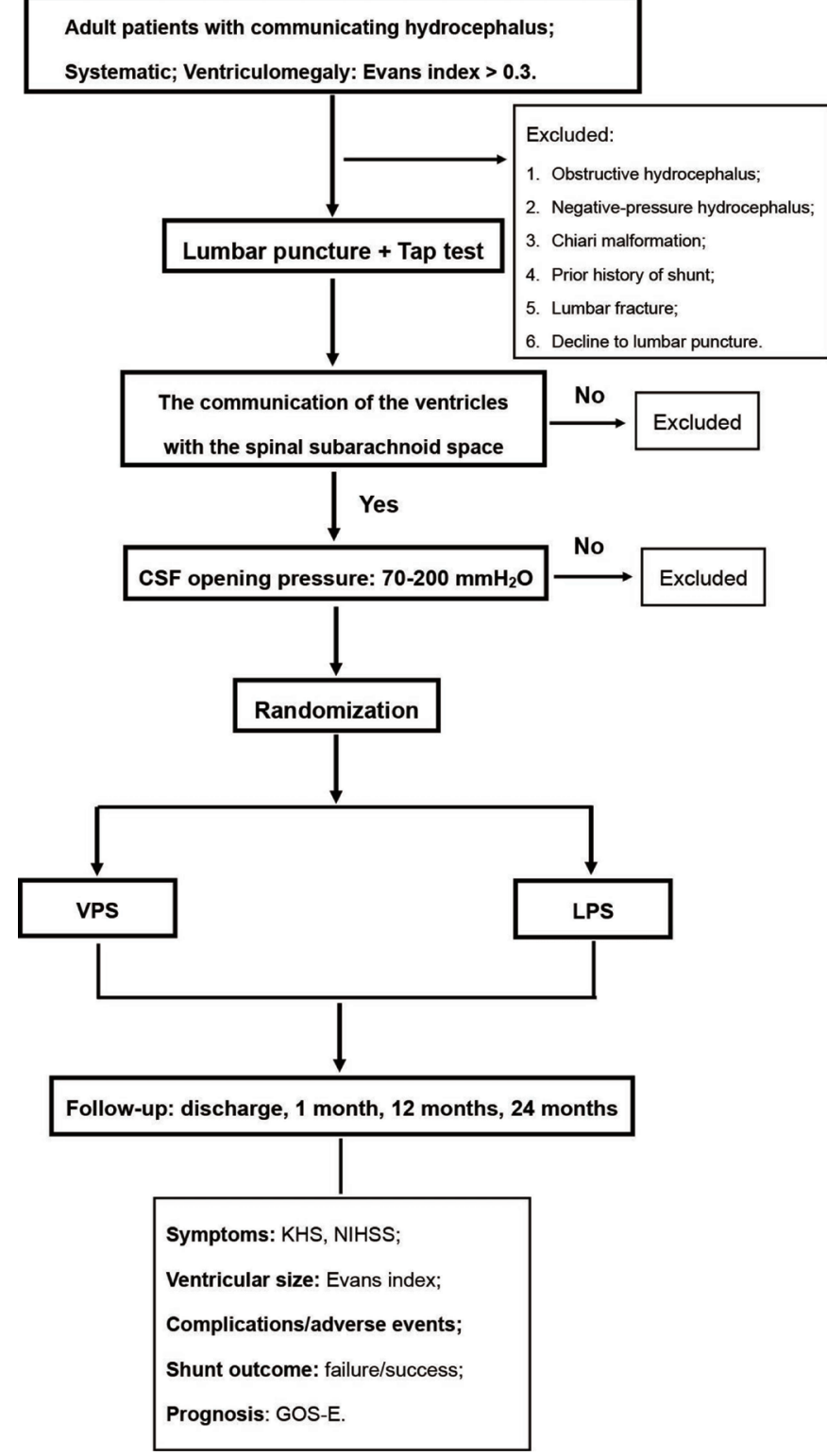

Figure 1 Flow chart of the selection of patients. CSF, cerebrospinal fluid; GOS-E, Glasgow Outcome Scale Extended; KHS, Keifer's Hydrocephalus Scal; LPS, lumboperitoneal shunt; NIHSS, National Institute of Health Stroke Scale; VPS, ventriculoperitoneal shunt.

and end in December 2025. Participants are recruited on outpatient department. Each participant will receive financial compensation. Specifically, once the eligible participants are admitted, three-dimension brain and spine MRI scan will be performed to further evaluate the ventricles, aqueduct, basal cisterns and spinal subarachnoid space, as well as to calculate the Evans index. Additionally, lumbar drainage is required to determine the eligibility.

\section{Inclusion criteria}

1. Age 18-90 years.

2. Symptomatic.

3. Communicating hydrocephalus.

4. Evans index $>0.3$. 
5. The communication of the ventricles with lumbar subarachnoid space is evident through lumbar puncture and CSF opening pressure is $70-200 \mathrm{~mm} \mathrm{H}_{2} \mathrm{O}$.

\section{Exclusion criteria}

1. Obstructive hydrocephalus.

2. Negative-pressure hydrocephalus.

3. Chiari malformation.

4. Prior history of shunt.

5. Lumbar fracture.

6. Decline to lumbar puncture.

\section{Randomisation and blinding}

Subjects who meet the inclusion criteria and sign the informed consents will be randomly allocated into one of two groups in a ratio of 1:1. The randomised allocation using a random number table will be conducted by a designated member who will not involve in other activities of study patients. The randomisation is not likely to blind for the subjects or attending neurosurgeons, but the data collectors, investigators and analysts are blinded.

\section{Intervention}

Neurosurgeons with extensive experience in the different procedures of CSF diversions will perform VPS or LPS, and will be trained centrally in advance and reach uniform standard. We will use the shunt system with programmable pressure valve, obtained from Medtronic (Minnesota, USA, LPS: 44421; VPS: 42866).

No matter which types of shunt system used, the initial pressure for the shunt system will set to the highest level before surgery. ${ }^{22}$ If patients had no improvement in clinical symptoms after surgery, we will check the shunt function and lower the pressure setting by one step, monitoring the safety of patients. $^{23}$

\section{Ventriculoperitoneal shunt}

The patients in the supine position receive general anaesthesia and then the head are turned to the left. A ventricular catheter is inserted into the lateral ventricle. A subcutaneous tunneler is made to connect the ventricles with abdominal cavity. The peritoneal catheter will be inserted if the CSF flow through shunt catheter is observed. The valve is placed at the cranial incision with a three-point fixation to the subcutaneous tissue.

\section{Lumboperitoneal shunt}

The patients in the left lateral position receive general anaesthesia and then the head are turned to the left. A lumbar catheter is inserted through the L3/4 or 2/3 interlaminar space into the spinal subarachnoid space. A subcutaneous flank region is then made to fix the valve. A subcutaneous tunneler is made to connect the spinal subarachnoid space, frank region and abdominal cavity. The peritoneal catheter will be inserted if the CSF flow through shunt catheter is observed.

\section{Outcomes}

Based on the study schedule (table 1), all patients will be analysed before shunt insertion, at the time of discharge, 1 month, 6 months, 12 months and 24 months postoperatively.

\section{Primary outcome}

The primary outcome measure is the rate of shunt failure at a 2-year follow-up term. On the basis of previous studies, shunt failure is defined as the occurrence of shunt revision owing to shunt obstruction, breakage, tubing exposure, malfunction, disconnection, infection or other conditions that require shunt revision. Shunt failure is also considered if improvement of symptoms or neurological function is not observed, corresponding to no improvement on the score of Keifer's Hydrocephalus Scale (KHS), National Institute of Health Stroke Scale (NIHSS) or Glasgow Outcome Scale Extended (GOS-E) within 2 years at evaluation point. Shunt success is defined as the lack of shunt failure.

\section{Secondary outcome}

The secondary outcomes include KHS, NIHSS, GOS-E, Evans index and safety endpoints, within 2 years after shunt implantation, as well as the cost-effectiveness of hospital stay. As shown in online supplemental files, KHS, a scale proposed by Kiefer, ${ }^{24}$ consists of five items: gait disturbances, mental disorder, urinary incontinence, headache and vertigo. The score of KHS ranges from 0 to 25 (higher is worse). The improvement of neurological function is evaluated by NHISS. A positive response to shunt implantation will be defined

\begin{tabular}{lcccc}
\hline Table 1 Study schedule & & & & \\
& Baseline & Discharge & 1 month & 12 months \\
\hline KHS & $\sqrt{ }$ & $\sqrt{ }$ & $\sqrt{ }$ & $\sqrt{ }$ \\
NIHSS & $\sqrt{ }$ & $\sqrt{ }$ & $\sqrt{ }$ & $\sqrt{ }$ \\
GOS-E & $\sqrt{ }$ & $\sqrt{ }$ & $\sqrt{ }$ & $\sqrt{ }$ \\
Evans index & $\sqrt{ }$ & $\sqrt{ }$ & $\sqrt{ }$ & $\sqrt{ }$ \\
Shunt outcomet & & $\sqrt{ }$ & $\sqrt{ }$ \\
Complications & & $\sqrt{ }$ & $\sqrt{ }$ \\
Mortality & & $\sqrt{ }$ & $\sqrt{ }$ \\
Cost-effectiveness & & $\sqrt{ }$ & & $\sqrt{ }$
\end{tabular}

*Evans index will be calculated through the MRI scan

t'Shunt outcome' includes shunt failure and shunt success.

GOS-E, Glasgow Outcome Scale Extended; KHS, Keifer's Hydrocephalus Scale; NIHSS, National Institute of Health stroke scale. 
as an improvement of more than one point in the KHS or NIHSS at evaluation point. The axial brain MRI scan is used to calculate the Evans index, which is the ratio of frontal horn to biparietal diameter.

Safety endpoint includes surgical complications, any adverse events and length of stay. The common complications after shunt surgery include overdrainage, intracranial haemorrhage, infection, malfunction, shunt obstruction, shunt migration, shunt disconnection, new epilepsy and abdominal symptoms. Severe adverse events (SAEs) refer to death, life-threatening events, shunt-related disability, hospitalisation for emergencies or intensive care unit or a prolonged hospitalisation period.

The cost-effectiveness of hospital stay will synchronously be investigated since the implanted system is not similar and the postoperative cost will be associated to the occurrence and management of complications.

\section{Data collection and management}

All patients will be analysed before shunt insertion, on the day of discharge, 1 month, 6 months, 12 months and 24 months postoperatively. At each site, two independent investigators will collect the baseline data such as age, gender, aetiology, date of admission, comorbidities, Glasgow Coma Scale, symptoms, KHS, NIHSS, Evans index, CSF parameters and CSF opening pressure. All patients will be followed up on a regular basis by outpatient visits. All data from hospitalisation and follow-up visits will be recorded in a paper-based table and then fixed into an electronic database. All data will be carefully examined and verified by these two investigators.

\section{Statistical analysis}

All data will be analysed using the statistical software program SPSS V.19 (IBM). Values of $\mathrm{p}<0.05$ (two sided) are considered to have statistical difference. For pairwise comparison, Bonferroni correction will be used, and the desired alpha level (0.05) divided by the number of comparisons equals the $p$ value required for significance. Categorical variables are statistically descried as number (per cent). We will use $\chi^{2}$ test to compare the difference on categorical variables (Fisher's exact test is used while appropriate). As referring to quantitative data, we will use Kolmogorov-Smirnov test is to determine the normality. If quantitative data follow normal distribution, described as arithmetic mean $\pm \mathrm{SD}$, we will use t-test to compare the difference. Other quantitative data will be described as median (range) and we will use Wilcoxon rank-sum test to compare the difference. Shunt-success rate curve is obtained using the method of Kaplan-Meier and logrank test is used to compare the difference between the two groups. The outcomes are presented as the incidence rate, or values, and its 95\% CIs, which will be calculated through SPSS program. We will use the Pearson's correlation to analyse the correlation between parameters.

\section{Data and safety monitoring}

We will set up a data monitoring committee (DMC) to guarantee the safety of this trial. All SAEs will be recorded in detail and reported to ethics committee. Members of the DMC will review all adverse events regularly, and hold a seminar to assess the risk and safety of the study if necessary.

\section{Data available statement}

The datasets generated and analysed during the current study are available from Research Manager (http://wwwmedresmanorgcn), as well as the corresponding author on reasonable request.

\section{DISCUSSION}

This study is currently the first randomised controlled trial comparing the two most commonly used techniques of shunt surgery (LPS and VPS) in the treatment of communicating hydrocephalus in order to provide high-level evidence. We believe that this trial is necessary since the benefits and harms to use one of these two methods are poorly understood. The results of the current study will provide high-level evidence for shunt-dependent hydrocephalus guidelines including the indications and contraindications to perform shunt, the standard procedures and the optimal option. This trial will also help to create an algorithm for the selection of suitable patients, preshunt and postshunt management.

Despite the potential strengths, there are some aspects of issues that need to be discussed. First, in this study, we will include symptomatic patients and asymptomatic patients will be excluded. The clinical manifestations of communicating hydrocephalus are various, such as gait/balance disturbance, dementia, urinary incontinence, headache, vertigo, psychiatric syndrome, etc. Patients with new or deteriorated symptoms that is estimated to be closely associated with hydrocephalus will be included in this trial. Elderly patients those who have at least one impairment of Hakim's triad and ventriculomegaly and are lack of known cause will be diagnosed as probably INPH, and Tap test, or external lumbar drainage, will be performed to determine the improvement of symptoms using KHS before allocation (online supplemental figure 1). The evaluation for INPH will help to differentiate with Alzheimer's disease and Parkinson's disease.

Second, KHS is chosen to evaluate the improvement of symptoms in this trial. Currently, there are no commonly accepted scales with the respect to the evaluation of symptoms for communicating hydrocephalus. However, there are a number of clinical scales widely used in patients with INPH such as INPH grading scale and Mini-Mental State Examination, which are focusing on the typical syndrome of INPH (gait/balance disturbance, dementia, urinary incontinence). ${ }^{10}$ Patients with communicating hydrocephalus are possible to develop various symptoms and signs. In this regard, KHS is a more appropriate scale since the five items of KHS are common symptoms for communicating hydrocephalus. ${ }^{132}$ Besides, the combination of KHS with NIHSS in this trial is probably superior to accurately evaluate the neurological symptoms and function.

Last, in terms of CSF opening pressure, we believe that pressures that are dramatically higher or lower than a range are likely not suitable for the upcoming LPS surgery. According to the Western guideline for the diagnosis of INPH,${ }^{25} \mathrm{CSF}$ 
opening pressure in the range of $70-245 \mathrm{~mm} \mathrm{H}_{2} \mathrm{O}$ is consistent with a probable NPH diagnosis but the range is suggested to be $<200 \mathrm{~mm} \mathrm{H}_{2} \mathrm{O}$ based on the Japanese guidelines for the diagnosis of INPH. ${ }^{26}$ In addition, there is no consensus in the optimal CSF opening pressure to perform LPS implantation. Taken together, a range of $70-200 \mathrm{~mm} \mathrm{H}_{2} \mathrm{O}$ is chosen in this study.

\section{ETHICS AND DISSEMINATION}

The study will be performed in compliance with the Declaration of Helsinki (2002) of the World Medical Association. The study was approved by Institutional Review Board of West China Hospital. All patients will be fully informed the potential benefits, potential risks and responsibilities, those who will sign the informed consents once they are included. Preliminary and final results will be published in peer-reviewed journals and presented at national and international congresses.

Contributors TS contributed to conceptualisation, methodology, quality assessment and writing original draft. WC and JY contributed to data curation, software and formal analysis. $Y Y$ and $Y Z$ contributed to investigation. $X L$ and HY contributed to formal analysis and writing original draft. CY contributed to conceptualisation, quality assessment and manuscript revision. JG contributed to conceptualisation, quality assessment, supervision, funding acquisition and manuscript revision. All authors approved the final manuscript.

Funding This trial is supported by West China Hospital of Sichuan University (No. 141191462).

Competing interests None declared.

Patient consent for publication Not required.

Provenance and peer review Not commissioned; externally peer reviewed.

Supplemental material This content has been supplied by the author(s). It has not been vetted by BMJ Publishing Group Limited (BMJ) and may not have been peer-reviewed. Any opinions or recommendations discussed are solely those of the author(s) and are not endorsed by BMJ. BMJ disclaims all liability and responsibility arising from any reliance placed on the content. Where the content includes any translated material, BMJ does not warrant the accuracy and reliability of the translations (including but not limited to local regulations, clinical guidelines, terminology, drug names and drug dosages), and is not responsible for any error and/or omissions arising from translation and adaptation or otherwise.

Open access This is an open access article distributed in accordance with the Creative Commons Attribution Non Commercial (CC BY-NC 4.0) license, which permits others to distribute, remix, adapt, build upon this work non-commercially, and license their derivative works on different terms, provided the original work is properly cited, appropriate credit is given, any changes made indicated, and the use is non-commercial. See: http://creativecommons.org/licenses/by-nc/4.0/.

ORCID iD

Junwen Guan http://orcid.org/0000-0002-4028-797X

\section{REFERENCES}

$1 \mathrm{Xu} \mathrm{H}$. New concept of the pathogenesis and therapeutic orientation of acquired communicating hydrocephalus. Neurol Sci 2016;37:1387-91.

2 Leinonen V, Vanninen R, Rauramaa T. Chapter 5 - Cerebrospinal fluid circulation and hydrocephalus. In: Kovacs GG, Alafuzoff I, eds. Handbook of clinical neurology. . Elsevier, 2018: 145. 39-50.

3 McAllister JP. Pathophysiology of congenital and neonatal hydrocephalus. Semin Fetal Neonatal Med 2012;17:285-94.
4 Kahle KT, Kulkarni AV, Limbrick DD, et al. Hydrocephalus in children. The Lancet 2016;387:788-99.

5 Wellons JC, Shannon CN, Holubkov R, et al. Shunting outcomes in posthemorrhagic hydrocephalus: results of a hydrocephalus clinical research network prospective cohort study. J Neurosurg Pediatr 2017;20:19-29.

6 Isaacs AM, Williams MA, Hamilton MG. Current update on treatment strategies for idiopathic normal pressure hydrocephalus. Curr Treat Options Neurol 2019;21:2019/12/03:65.

7 Reddy GK, Bollam P, Caldito G. Long-term outcomes of ventriculoperitoneal shunt surgery in patients with hydrocephalus. World Neurosurg 2014;81:404-10.

8 Gholampour S, Bahmani M. Hydrodynamic comparison of shunt and endoscopic third ventriculostomy in adult hydrocephalus using in vitro models and fluid-structure interaction simulation. Comput Methods Programs Biomed 2021;204:106049.

9 Gholampour S. Fsi simulation of CSF hydrodynamic changes in a large population of non-communicating hydrocephalus patients during treatment process with regard to their clinical symptoms. PLoS One 2018;13:e0196216.

10 Pinto FCG, Saad F, Oliveira MFde, MFd O, et al. Role of endoscopic third ventriculostomy and ventriculoperitoneal shunt in idiopathic normal pressure hydrocephalus: preliminary results of a randomized clinical trial. Neurosurgery 2013;72:845-54.

11 Kazui $\mathrm{H}$, Miyajima M, Mori E, et al. Lumboperitoneal shunt surgery for idiopathic normal pressure hydrocephalus (SINPHONI-2): an openlabel randomised trial. Lancet Neurol 2015;14:585-94.

12 Sun T, Yuan Y, Zhang Q, et al. One-Year outcome of patients with posttraumatic hydrocephalus treated by lumboperitoneal shunt: an observational study from China. Acta Neurochir 2018;160:2031-8.

13 Sun T, Yuan Y, Zhang Q, et al. Establishing a preoperative evaluation system for Lumboperitoneal shunt: approach to attenuate the risk of shunt failure. World Neurosurg 2018;117:e308-15.

14 Wang Z, Wang K, Qian Z, et al. Lumboperitoneal and ventriculoperitoneal shunt surgery for posthemorrhagic communicating hydrocephalus: a comparison. World Neurosurg 2019;127:e638-43.

15 Chen Q, Feng Z, Tan Q, et al. Post-hemorrhagic hydrocephalus: recent advances and new therapeutic insights. J Neurol Sci 2017;375:220-30.

16 Sun T, Li X, Zhang Q, et al. Efficacy and safety of Lumboperitoneal shunt in the treatment of all-cause communicating hydrocephalus: analysis of risk factors of shunt failure. World Neurosurg 2019;132:e956-62.

17 Wang VY, Barbaro NM, Lawton MT, et al. Complications of lumboperitoneal shunts. Neurosurgery 2007;60:1045-9.

18 Miyajima M, Kazui H, Mori E, et al. One-year outcome in patients with idiopathic normal-pressure hydrocephalus: comparison of lumboperitoneal shunt to ventriculoperitoneal shunt. J Neurosurg 2016;125:1483-92.

19 Giordan E, Palandri G, Lanzino G, et al. Outcomes and complications of different surgical treatments for idiopathic normal pressure hydrocephalus: a systematic review and meta-analysis. J Neurosurg 2019;131:1024-36.

20 Chan A-W, Tetzlaff JM, Altman DG, et al. Spirit 2013 statement: defining standard protocol items for clinical trials. Ann Intern Med 2013;158:200-7.

21 Giordan E, Palandri G, Lanzino G, et al. Outcomes and complications of different surgical treatments for idiopathic normal pressure hydrocephalus: a systematic review and meta-analysis. J Neurosurg 2018:1-13.

22 Nakajima M, Miyajima M, Akiba C, et al. Lumboperitoneal shunts for the treatment of idiopathic normal pressure hydrocephalus: a comparison of Small-Lumen abdominal catheters to gravitational add-on valves in a single center. Oper Neurosurg 2018;15:634-42.

23 Marupudi NI, Harris C, Pavri T, et al. The role of lumboperitoneal shunts in managing chronic hydrocephalus with slit ventricles. $J$ Neurosurg Pediatr 2018;22:632-7.

24 Meier U, Kiefer M, Sprung C. Evaluation of the Miethke dual- switch valve in patients with normal pressure hydrocephalus. Surg Neurol 2004;61:119-27.

25 Relkin N, Marmarou A, Klinge P, et al. Diagnosing idiopathic normalpressure hydrocephalus. Neurosurgery 2005;57:S2-4-S2-16.

26 Mori E, Ishikawa M, Kato T, et al. Guidelines for management of idiopathic normal pressure hydrocephalus: second edition. Neurol Med Chir 2012;52:775-809. 